\title{
MISTRAL: A NEW PROGRAM FOR PRECISE ATOMIC MASS DETERMINATIONS OF NUCLIDES FAR FROM STABILITY
}

\author{
M.D. Lunney, G. Audi, C. Borcea, M. Dedieu, H. Doubre, M. Duma, M. Jacotin, \\ J.-F. Képinski, G. Le Scornet, M. de Saint Simon and C. Thibault \\ Centre de Spectrométrie Nucléaire et de Spectrométrie de Masse \\ (CSNSM-IN2P3-CNRS, F-91405 Orsay, France) \\ and the MISTRAL collaboration*)
}

\begin{abstract}
:
The MISTRAL project (Mass measurements at ISOLDE using a Transmission Radio frequency spectrometer on-Line) is scheduled to begin experiments towards the end of 1996. With high resolution $\left(10^{5}\right)$, potentially high accuracy $\left(5 * 10^{-7}\right)$ and excellent sensitivity $\left(10 \mathrm{~s}^{-1}\right)$, the MISTRAL spectrometer promises to provide needed mass measurements in regions of very-short lived nuclei. The spectrometer operation principles are described and a discussion concerning the interest of using highly charged ions is presented.

Presented at the 1st Euroconference on Atomic

Physics with stored highly-charged ions, Heidelberg, Germany, 1995; submitted to Hyperfine Interactions
\end{abstract}

(IS 346)

*) The Mistral Collaboration:

CSNSM, Orsay; IAP, Bucharest; PPE Division, CERN; GSI, Darmstadt; University of Mainz; McGill University, Montreal; IPN, Orsay; Chalmers University, Gothenburg; University of Giessen and the ISOLDE Collaboration 



\title{
MISTRAL: A new program for precise atomic mass determinations of nuclides far from stability
}

\author{
M.D. Lunney, G. Audi, C. Borcea, M. Dedieu, H. Doubre, M. Duma, M. Jacotin, \\ J.-F. Képinski, G. Le Scornet, M. de Saint Simon and C. Thibault \\ Centre de Spectrométrie Nucléaire et de Spectrométrie de Masse \\ (CSNSM-IN2P3-CNRS, F-91405 Orsay, France) and the MISTRAL collaboration ${ }^{\S}$
}

\begin{abstract}
The MISTRAL project (Mass measurements at ISolde using a Transmission RAdiofrequency spectrometer on-Line) is scheduled to begin experiments towards the end of 1996. With high resolution $\left(10^{5}\right)$, potentially high accuracy $\left(5 \times 10^{-7}\right)$ and excellent sensitivity $\left(10 \mathrm{~s}^{1}\right)$, the MISTRAL spectrometer promises to provide needed mass measurements in regions of very-short lived nuclei. The spectrometer operation principles are described and a discussion concerning the interest of using highly charged ions is presented.
\end{abstract}

\section{Introduction}

The precise determination of atomic masses has important ramifications for metrology, atomic physics, weak interaction physics as well as nuclear structure and stellar nucleosynthesis [1,2]. The latter two cases require accurate measurements of radioisotopes very far from stability in order to provide stringent tests for nuclear mass models. This allows the elucidation of new nuclear structure and dependable predictions for masses and other nuclear properties for nuclei at the limits of particle stability and beyond. Reliable predictions are crucial for stellar nucleosynthesis scenarios as some of the processes involve nuclides that may never be produced in the laboratory.

Penning traps offer the most accurate mass determinations by measuring the cyclotron frequency of ions confined for long periods in a very homogenous magnetic field. This providesvery high resolution with accuracies better than one part in $10^{10}[3,4]$. Even better resolution is possible by using highly charged ions due to their correspondingly higher cyclotron frequency [5]. However, these measurements are made using stable ionic species. While the antiproton mass measurement at LEAR, CERN [6] has provided a test of CPT invariance to one ppb using a Penning trap, the ISOLTRAP program at CERN [7], has now brought the power of the Penning trap to nuclear physics with measurements of unstable $\mathrm{Cs}, \mathrm{Sr}, \mathrm{Rb}$ and Fr isotopes to the level of $0.1 \mathrm{ppm}$ including the first resolution of an isomeric state by mass spectrometry [8]. A new Penning trap system is currently being developed at Chalk River Nuclear Laboratories in Canada for mass measurements using laser-desorbed radioactive ions [9].

\footnotetext{
$\S$ CSNSM, Orsay; IAP, Bucharest; PPE Division, CERN; GSI, Darmstadt; University of Mainz; McGill University,
} Montreal; IPN, Orsay; Chalmers University, Göteborg; University of Giessen and the ISOLDE Collaboration. 
In general, masses of unstable isotopes are measured using nuclear reactions and decays ( $Q$ values) and by various techniques of direct mass spectrometry at high and low energies. While some decay methods, $\mathrm{Q}_{\alpha}$ measurements for example, produce very accurate mass differences, they can be somewhat prone to systematic error if not closely linked to a well-known absolute value. Furthermore, incomplete knowledge of the decay scheme, especially in the case of $\mathrm{Q}_{\beta}$ measurements, can have adverse effects. Mass spectrometry generally provides a series of systematic and somewhat independent measurements performed with accompanying calibrations. These measurements are carried out "on line" at isotope- or fragment-separator facilities where the ions to be measured are transported to the spectrometer as a beam. Such measurements are ultimately limited by the production yield of the separator facility combined with the sensitivity of the apparatus. Several mass spectrometry programs exist at various facilities. Of these, only one still uses electro/magnetostatic techniques and is complemented by radioactivity identification [10]. Other experiments determine the mass from time-of-flight, such as TOFI at Los Alamos [11], SPEG at GANIL [12], and a novel technique developed at GANIL that lengthens the flight time using the multiple turns of ions in a cyclotron [13]. Finally, some methods rely on the inherently accurate measurement of the cyclotron frequency, as in the Penning trap systems mentioned above. Also, a new program at GSI provides high accuracy mass determinations using the Schottky pickup signal of ions circulating in the ESR storage ring [14].

\section{The MISTRAL Spectrometer}

A new mass measurement project, MISTRAL, has recently been approved at ISOLDE which uses a radiofrequency (or Smith-type) transmission spectrometer to determine the cyclotron frequency of an ion during a short trajectory in a homogeneous magnetic field [15]. MISTRAL will complement ISOLTRAP by concentrating on the very short-lived nuclei that become more difficult to measure accurately in the Penning trap due to the long storage times that are required.

A schematic diagram of the ion trajectory envelope in the MISTRAL spectrometer is shown in figure 1. Ions are injected into the spectrometer and vertically deflected so that they make two helicoidal turns inside the magnetic field. The principle of the measurement technique is the determination of the cyclotron frequency using two successive modulations of the ion kinetic energy. Ions for which the net modulation is zero - the resonant condition - are transmitted through a narrow slit after the final turn. High resolution is obtained by performing this modulation with a radiofrequency voltage. The ions are exactly demodulated after one turn if the modulation frequency $\omega_{\mathrm{RF}}$ satisfies the following relationship:

$$
\omega_{\mathbf{R F}}=[\mathbf{n}+1 / 2] \omega_{\mathbf{c}}
$$

where $n$ is an integer harmonic and $\omega_{c}$ is the cyclotron frequency of an ion of mass $m$ and charge $q$ in the magnetic field $\boldsymbol{B}$ given by: $\omega_{c}=q \boldsymbol{B} / \mathrm{m}$. This idea is illustrated in figure 2, for which the value 
$n=1$ is taken as an example. Thus the spectrometer is used as a type of clock that determines the slower cyclotron frequency using the faster radiofrequency. The value of $n$ determines the resolving power of the instrument $R$, given a certain diameter modulation $D_{m}$ and slit size $w$, according to the relationship:

$$
\mathbf{R}=\mathbf{2} \pi \mathbf{n} \frac{\mathbf{D}_{\mathbf{m}}}{\mathbf{w}}
$$

The RF is scanned, resulting in the detected signal shown in figure 3 for $n=1500$ which shows a resolving power of over 500,000. This signal was recorded with a slit size of $0.2 \mathrm{~mm}$ and a $1 \%$ amplitude modulation of a $200 \mathrm{kV}$ beam of $\mathrm{H}^{-}$ions.

The layout of the spectrometer, as foreseen at ISOLDE, is shown in figure 4. A reference ion will be injected alternately with the unknown mass from the ISOLDE beamline to avoid systematic error due to long term drifts of the magnetic field. The unknown mass is then determined from the reference mass from the ratio of their measured cyclotron frequencies.

\section{Potential MISTRAL Performance}

\section{A: Initial configuration}

The MISTRAL spectrometer is currently being adapted for measuring over the wide range of masses produced on-line at ISOLDE. The spectrometer acceptance has been enlarged using slits $0.4 \mathrm{~mm}$ wide by $10 \mathrm{~mm}$ high. When this acceptance is compared with the measured ISOLDE emittance of $32 \pi \mathrm{mm}$ mrad, a transmission of about $1 \%$ is obtained. Taking into account the background of the detector, a sensitivity of about 10 atoms/s can be expected. The modulation amplitude has been chosen as $1 \%$, requiring an RF voltage of about $300 \mathrm{~V}$ for the $60 \mathrm{kV}$ ISOLDE beam. The resolving power of the spectrometer, in this configuration, will be greater than $10^{5}$. This allows good transmission and is sufficient for eliminating isobaric contaminants. The expected accuracy requires the evaluation of three contributions: the error resulting from the instability of the magnetic field, the statistical error, and the systematic error. The magnetic field variation has been measured as $5 \times 10^{-8}$ on a time scale of some seconds. Assuming 500 counts in the peak, the statistical error is $2 \times 10^{-7}$. The systematic error has yet to be evaluated but we estimate an overall accuracy of $5 \times 10^{-7}$ (i.e., $50 \mathrm{keV}$ for $A=100$ ) in most cases.

Being a transmission spectrometer, a consequence of measuring the mass of a certain isotope is that the detected ions are effectively a purification of the incident beam. This enables the detection region to be used for auxiliary measurements such as nuclear spectroscopy to study decay modes and nuclear lifetimes on isobarically-pure samples. In cases where isomeric mixtures are present, it is intended to perform such complementary spectroscopy in order to determine the relative intensities and lifetimes to accurately fit the detected mass peak. 
Since the time of the measurement is only limited by the time-of-flight through the spectrometer (about $50 \mu \mathrm{s}$ ), the limit on measurable nuclear half-life will be determined by the release time of the ISOLDE target. Furthermore, the measurement procedure is direct and universal, with no collection or secondary ionization schemes necessary.

\section{B: Incident Beam Cooling}

As the spectrometer transmission depends on the slit size (and therefore, the resolution), it is important to have a small emittance. In a second phase of operation it is planned to incorporate a beam cooling stage immediately preceding the spectrometer in order to reduce the emittance of the incident beams. This beam cooler could take the form of either a Paul trap [16] or a quadrupole mass filter using collisional focusing [17]. Current estimates from work developing Paul traps and mass filters as beam collectors and coolers [18] indicate that a reduction in beam emittance of an order of magnitude may be possible. This means that, with the corresponding increase in transmission, the spectometersensitivity would be such that measurements of nuclides produced with yields of 1 atom/s would be possible. Alternately, the exit slit size could be reduced from the present $0.4 \mathrm{~mm}$ enabling higher resolution which would be useful for isomeric mixtures. An added advantage of a cooler is the elimination of any systematic error contributions due to the differing origins of the reference and unknown ions.

\section{C: The Interest of highly charged ions for the MISTRAL project}

While the MISTRAL spectrometer has been designed to cover practically the entire ISOLDE mass range, the separator voltage must be reduced in order to accomodate the heaviest isotopes within the linear range of the spectrometer's magnetic field. The spectrometer operating parameters corresponding to four different mass ranges are listed in table 1.

For heavier ions to circulate in the spectrometer at the fixed radius $r$ (of $0.5 \mathrm{~m}$ ), the magnetic field, $B$ must be increased to compensate for the higher mass $m$, according to the following relation:

$$
\mathbf{B}=\frac{1}{\mathbf{r}} \sqrt{\frac{2 \mathrm{Vm}}{\mathrm{q}}}
$$

However, at a certain point, the field saturates and in order to conserve the fixed radius, the ion acceleration potential, $V$ must be reduced, as seen in table 1. In principle, this poses no major problems however, in reality, the increased emittance of the beam will not only make separator adjustment difficult, but also deteriorate the sensitivity of the spectrometer. An alternative to this situation would be to increase the charge state, $q$ in order to keep the field within its design value and in turn, preserve the nominal value of the accelerator voltage.

Another important fact, relevant for adapting the spectrometer for use over a large mass range, is that the RF modulator geometry depends on the ion velocity inside the spectrometer. This 
explains the fact that for each of the four mass ranges shown in table 1, there is a corresponding modulator geometry consisting of the accelerating gaps, $g$ and the electrode drift length, $d$ as shown in figure 4 (lower right). The gaps are made as small as possible to maximize the uniformity of the modulating field whereas the drift length depends on the ion velocity as well as the RF frequency. Since two ground electrodes are needed to properly define the modulating field, each modulation is a result of the ion crossing two gaps. In order to ensure that the two accelerations do not cancel each other in the first passage through the modulator, $d$ must be long enough to let at least one RF cycle elapse before the ion reaches the second gap. This idea is illustrated in figure 5. The sum of $d$ and $g$ is equal to $v(k+1 / 2) / f_{R F}$ where $v$ is the ion velocity and $k$ is an integer which should be as small as possible to conserve isochronism. Higher modes of $k$ correspond to a longer distance that allows the RF to oscillate through more cycles, as shown in figure 5.

By using ions of higher charge states, it is possible to reduce the number of modulator geometries to one. This would greatly increase the versatility of the spectrometer as no intervention would be required between measurements in differing mass ranges. Listed in table 2 are the corresponding charge states necessary for one modulator geometry of $g=0.5 \mathrm{~mm}$ and $d=3.36 \mathrm{~mm}$. Note also that the magnetic field is kept within its linear operating range and the ISOLDE separator voltage is maintained at its nominal operating value for each mass range.

The question naturally arises as to the source of such highly charged ions as there is no capacity for such in the ISOLDE source. The answer could be found in another recently approved experiment called REX-ISOLDE (Radioactive beam EXperiments at ISOLDE) [19]. This experiment will include a Penning trap beam collection and cooling stage followed by an electron beam ion source (EBIS) that will provide high charge states (up to 4) for isotopes produced by the ISOLDE target. REX will then post-accelerate these ions to perform coulomb excitation and neutron transfer experiments. The proposal also includes the possiblity of a switchyard at the output of the EBIS in order to furnish highly charged radioactive ions for other experiments [19,20]. The EBIS could also be used to provide the reference mass as well, eliminating any systematic error due to different ion sources for the measurement.

As outlined in the REX proposal [19], the overall efficiency of the charge state breeding stage should be greater than $10 \%$ and the total collection and breeding time less than $50 \mathrm{~ms}$. The time delay is not too serious as it is the same order of magnitude as the ISOLDE release time meaning that isotopes with much shorter lifetimes will not diffuse out of the target in any case. Furthermore, the cooling process in the Penning trap is highly mass selective so that the beam will be greatly purified. On the other hand, the loss of $90 \%$ of the beam in charge state breeding will have to be balanced against the gains made using highly charged ions. Rather than definitively improving spectrometer performance, highly charged ions would make it more adaptable. 


\section{Conclusion}

The MISTRAL experiment will commence its measurement program at ISOLDE in late 1996. MISTRAL promises to provide a wealth of new and accurate mass data for very short-lived isotopes. This will contribute towards the improvement of nuclear mass models with the ultimate goal of reliably predicting masses and other properties of nuclides located on the astrophysical stellar nucleosynthesis paths and out to the limits of nuclear particle stability.

There are more than 50 radionuclides produced at rates greater than 10 atoms/s (according to the older ISOLDE SC yields) and with half-lives of less than one second for which no measured mass values exist. Another 50 isotopes exists for which the mass values can be significantly improved, representing a healthy long-term measurement program for MISTRAL.

In a second phase, an intermediate incident beam cooling stage is planned in order to augment the spectrometer sensitivity or resolution. There are also significant potential benefits for the program if a source of highly charged ions were made available.

\section{References}

[1] Proceedings of the Nobel Symposium 91: Trapped charged particles and related fundamental physics, Lysekil, Sweden, 1994, to appear in Physica Scripta, 1995.

[2] G. Audi and A.H. Wapstra, in [1] and references therein.

[3] R. S. Van Dyck, Jr., D. L. Farnham, and P. B. Schwinberg, Phys. Rev. Lett. 19 (1993) 2888; R.S. Van Dyck, Jr. et al., in [1].

[4] E.A. Cornell et al., Phys. Rev. Lett. 63 (1989) 1674; D. Pritchard et al., in [1].

[5] T. Schwartz et al., these proceedings; C. Carlberg et al., in [1].

[6] G. Gabrielse et al., Phys. Rev. Lett. 74 (1995) 3544.

[7] H. Stolzenberg et al., Phys. Rev. Lett. 65 (1990) 3104; G. Bollen et al., submitted to Nucl. Instr. Meth. in Phys. Res. A, 1995.

[8] G. Bollen et al., Phys. Rev. C 46 (1992) R2140.

[9] K.S. Sharma et al., Hyp. Int. 81 (1993) 217.

[10] K.S.Sharma et al., Phys. Rev. C 44 (1991) 2439.

[11] H.L. Seifert et al., Z. Phys. A 349 (1994) 25 and references therein.

[12] N.A. Orr, et al., Phys. Lett. B 258 (1991) 29 and references therein.

[13] G. Auger et al., Nucl. Instr. and Meth. in Phys. Res. A 350 (1994) 235-243.

[14] B. Schlitt et al., these proceedings; H. Geissel et al., Phys. Rev. Lett. 68 (1992) 3412.

[15] M. de Saint Simon et al., in [1]; M. de Saint Simon et al., Nucl. Instr. Meth. in Phys. Res. B70 (1992) 459.

[16] R.B. Moore and M.D. Lunney, in: Practical Aspects of Ion Trap Mass Spectrometry, eds. R.E. March and J.F.J. Todd, vol. 2 (CRC Press, Boca Raton, 1995).

[17] D.J. Douglas and J.B. French, J. Am. Soc. Mass Spectrom. 3 (1992) 398.

[18] R.B. Moore et al., in [1]. 
[19] D. Habs, REX-ISOLDE proposal, CERN/ISC 94-25 (1994).

[20] P. Van Duppen, private communication (1995). 


\section{Figure Captions}

Figure 1: Schematic view of the ion trajectory envelope in the radiofrequency mass spectrometer. The ions follow two turns in the magnetic field: injection of one half turn, modulation and demodulation over one turn, and extraction through a narrow slit after the final half turn.

Figure 2: Relationship between the cyclotron frequency (represented by a projection of the ion displacement onto the diameter of the trajectory) and the RF modulating voltage of ions in both resonant and non-resonant cases. In this example, we take $n=1$ in equation (1) such that $\omega_{\mathrm{rf}}=3 / 2 \omega_{\mathrm{c}}$ for the resonant condition. The effects of the kinetic energy modulations are such that, in resonance (middle), the modulation and demodulations (dashed lines) over one cyclotron orbit cancel whereas out of resonance (below), the ions deviate from the fixed trajectory as the net modulation is not zero.

Figure 3: Detected signal of a $200 \mathrm{kV}$ beam of $\mathrm{H}^{-}$ions transmitted through the radiofrequency spectrometer as a function of modulating frequency showing the peak corresponding to the harmonic $n=1500$. The data are fit to a symmetric triangular peak yielding a FWHM of better than 500,000.

Figure 4: Overhead schematic view of the MISTRAL spectrometer with the detail of the modulator shown at lower right. The unknown mass is determined by alternately injecting a calibration mass from a reference ion source and comparing the cyclotron frequencies. A detection chamber located after the final slit also serves to perform nuclear spectroscopy on the transmitted (filtered) ion beam.

Figure 5: Relationship between the modulator geometry (top) and the RF voltage signal. The drift length, $d$ is calculated (based on the ion velocity) such that the ion experiences a net acceleration over the two field gaps shown by the dashed lines. (middle) case where the RF oscillates over one cycle (mode $k=1$ ) and (below) where the RF oscillates over two cycles (mode $k=2$ ).

\section{Table Captions}

Table 1: Mass range of the RF mass spectrometer with the corresponding ISOLDE acceleration voltage $V$, and MISTRAL operating parameters for the magnetic field $B$, the corresponding cyclotron frequency $f_{c}$, and corresponding harmonic number $n$, giving a resolution of at least 100,000 . The last three columns describe the modulator geometry (see text).

Table 2: $\quad$ MISTRAL operating parameters and the corresponding charge state $q$ required to cover the indicated mass range with a single modulator geometry $(g=0.5 \mathrm{~mm}$ and $d=3.36 \mathrm{~mm})$. The ISOLDE separator voltage does not change and the magnetic field is well within its linear region of 0.1-0.9 T. Note that to keep within the bandwidth of the RF amplifier $(250-500 \mathrm{MHz})$ the modulation 
frequency for the first two cases corresponds to the modulator mode $k=1$ and the last two, to $k=2$. 


\begin{tabular}{|c||c|c|c||c|c||c||c||}
\hline \hline mass & $V(\mathrm{kV})$ & $\boldsymbol{B}(\mathrm{T})$ & $f_{C}(\mathrm{kHz})$ & $n$ & $g(\mathrm{~mm})$ & $d(\mathrm{~mm})$ & $k$ \\
\hline \hline $7-28$ & 60 & $0.2-0.4$ & $410-205$ & 1220 & 0.5 & 3.36 & 1 \\
\hline $23-92$ & 60 & $0.3-0.7$ & $225-115$ & 2212 & 0.5 & 1.63 & 1 \\
\hline $84-200$ & $60-40$ & $0.6-0.8$ & $120-60$ & 4230 & 0.4 & 1.46 & 2 \\
\hline $170-250$ & $45-30$ & 0.8 & $70-50$ & 5160 & 0.4 & 1.13 & 2 \\
\hline
\end{tabular}

\begin{tabular}{|c||c||c|c|c||c|c||}
\hline \hline mass & $V(\mathrm{kV})$ & $\boldsymbol{B}(\mathrm{T})$ & $f_{C}(\mathrm{kHz})$ & $f_{R F}(\mathrm{MHz})$ & $n$ & $q$ \\
\hline \hline 10 & 60 & 0.22 & 343 & 418 & 1220 & 1 \\
\hline 40 & 60 & 0.32 & 242 & 296 & 1220 & 2 \\
\hline 160 & 60 & 0.52 & 148 & 302 & 2035 & 3 \\
\hline 260 & 60 & 0.57 & 134 & 273 & 2035 & 4 \\
\hline
\end{tabular}




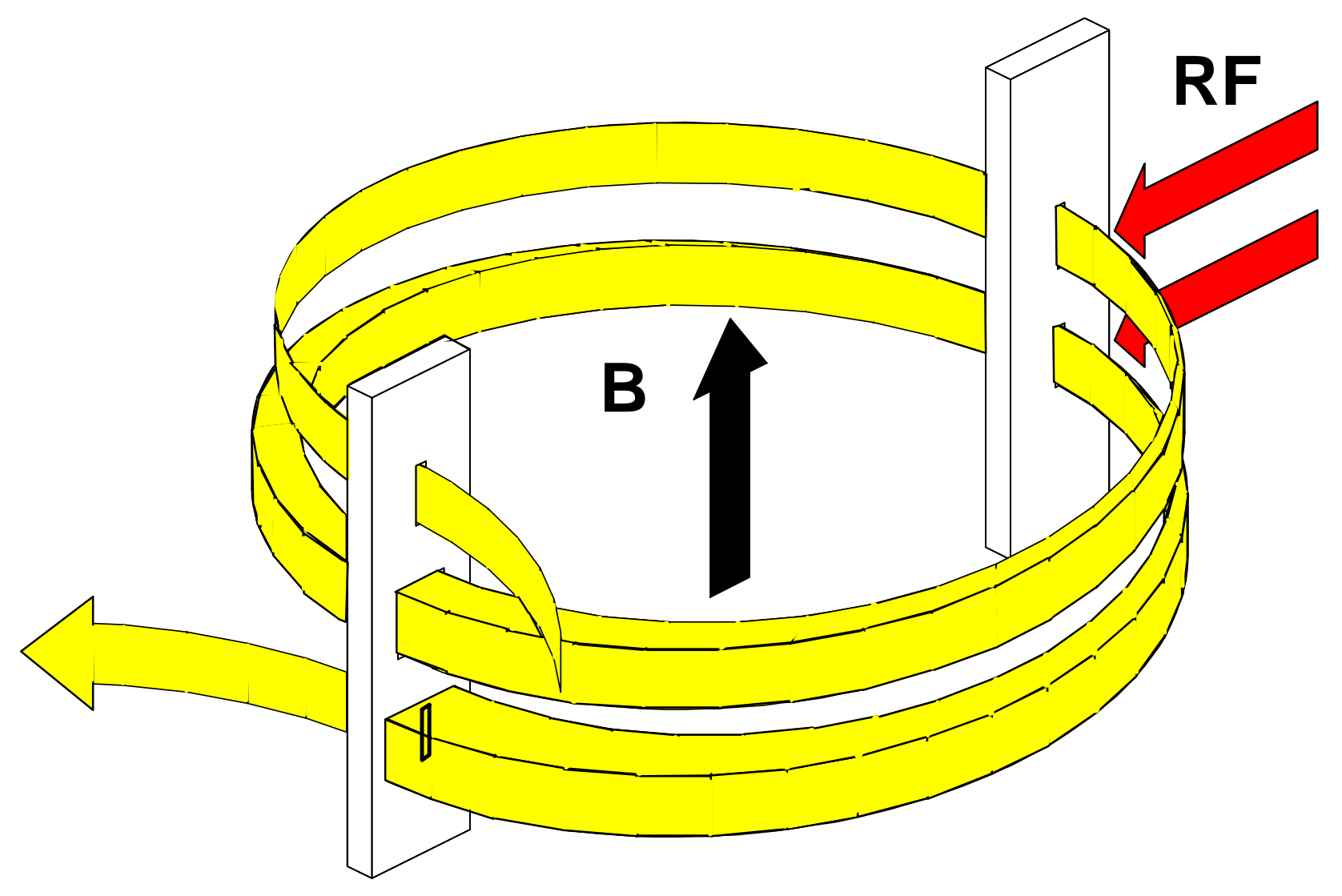




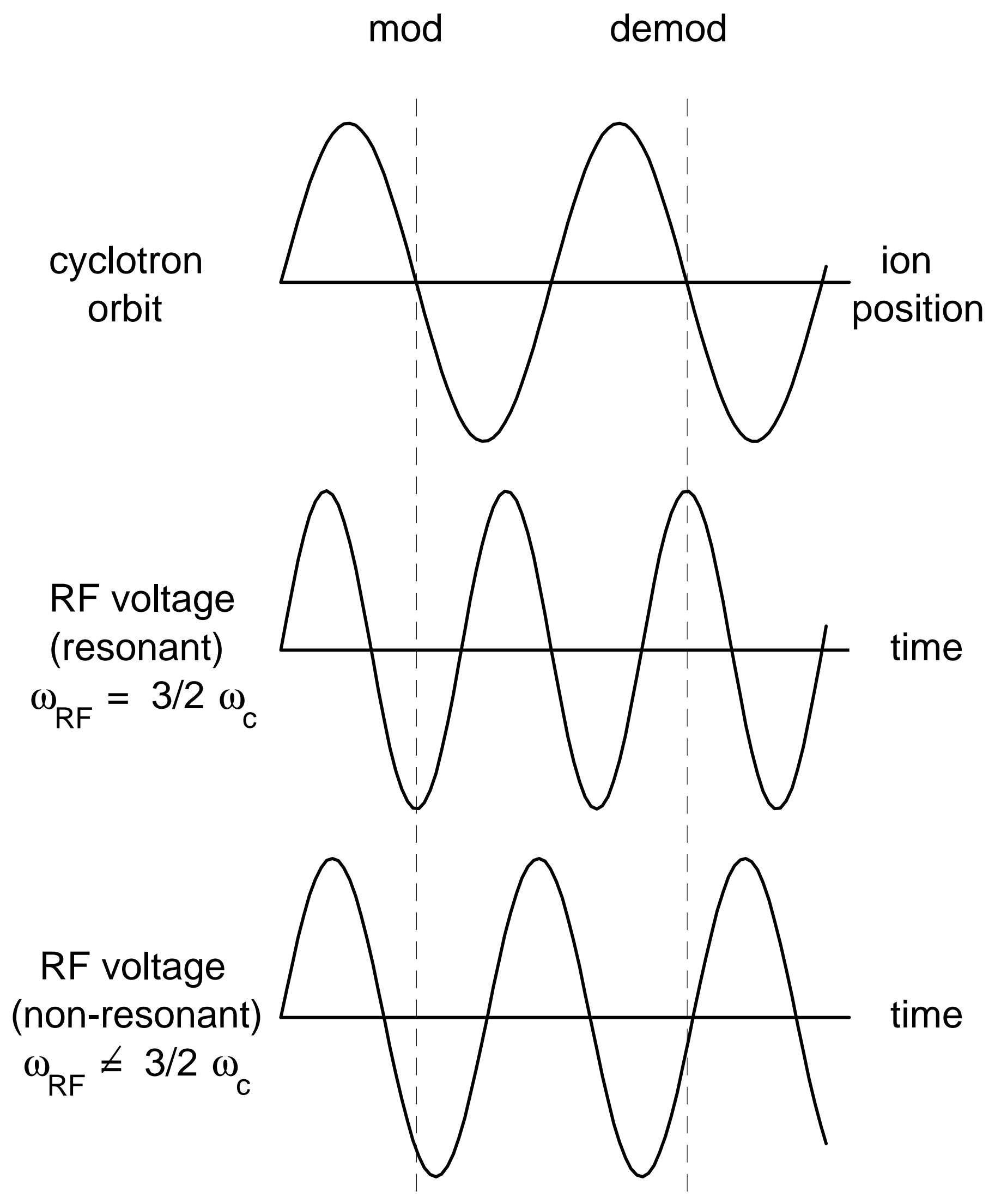




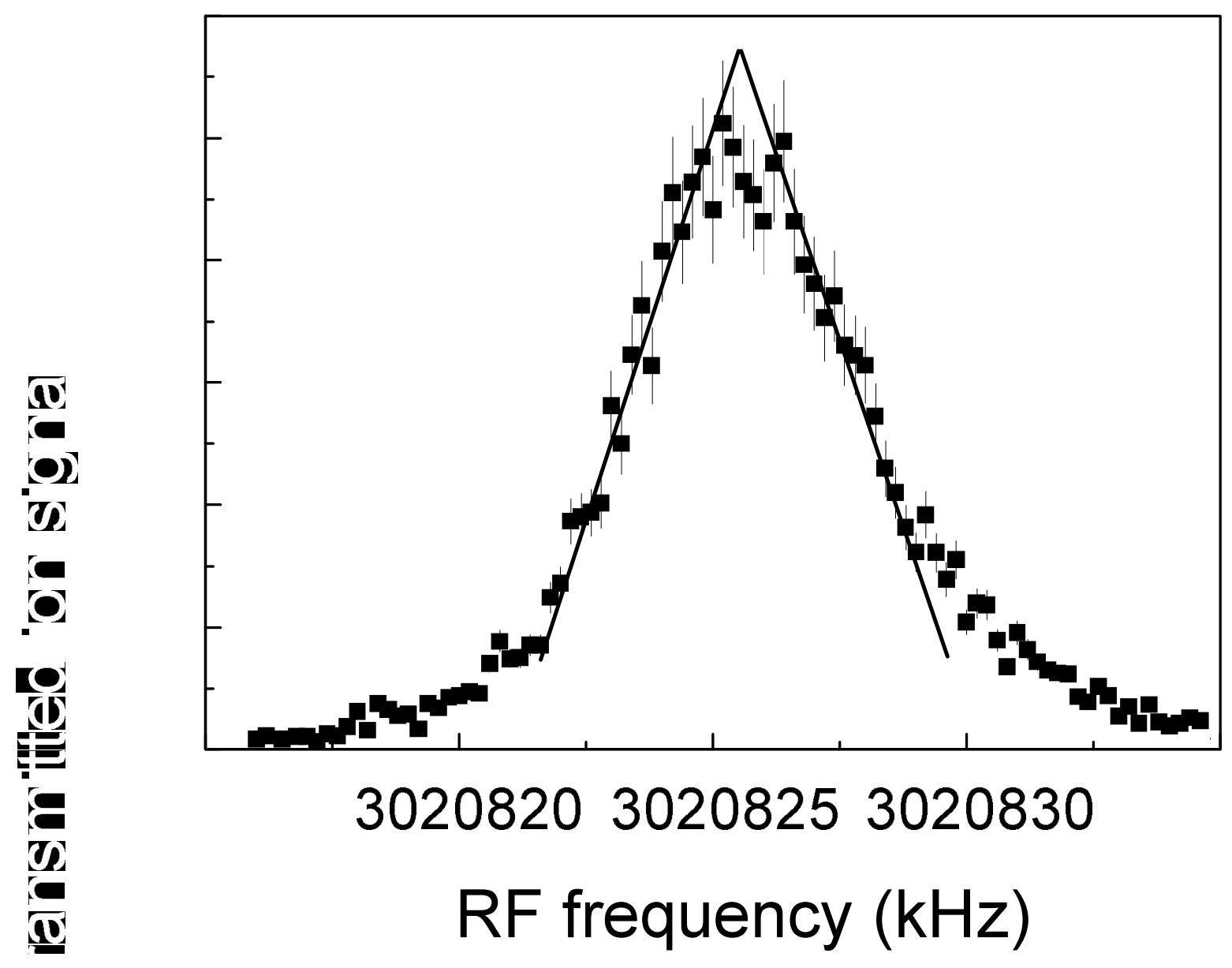




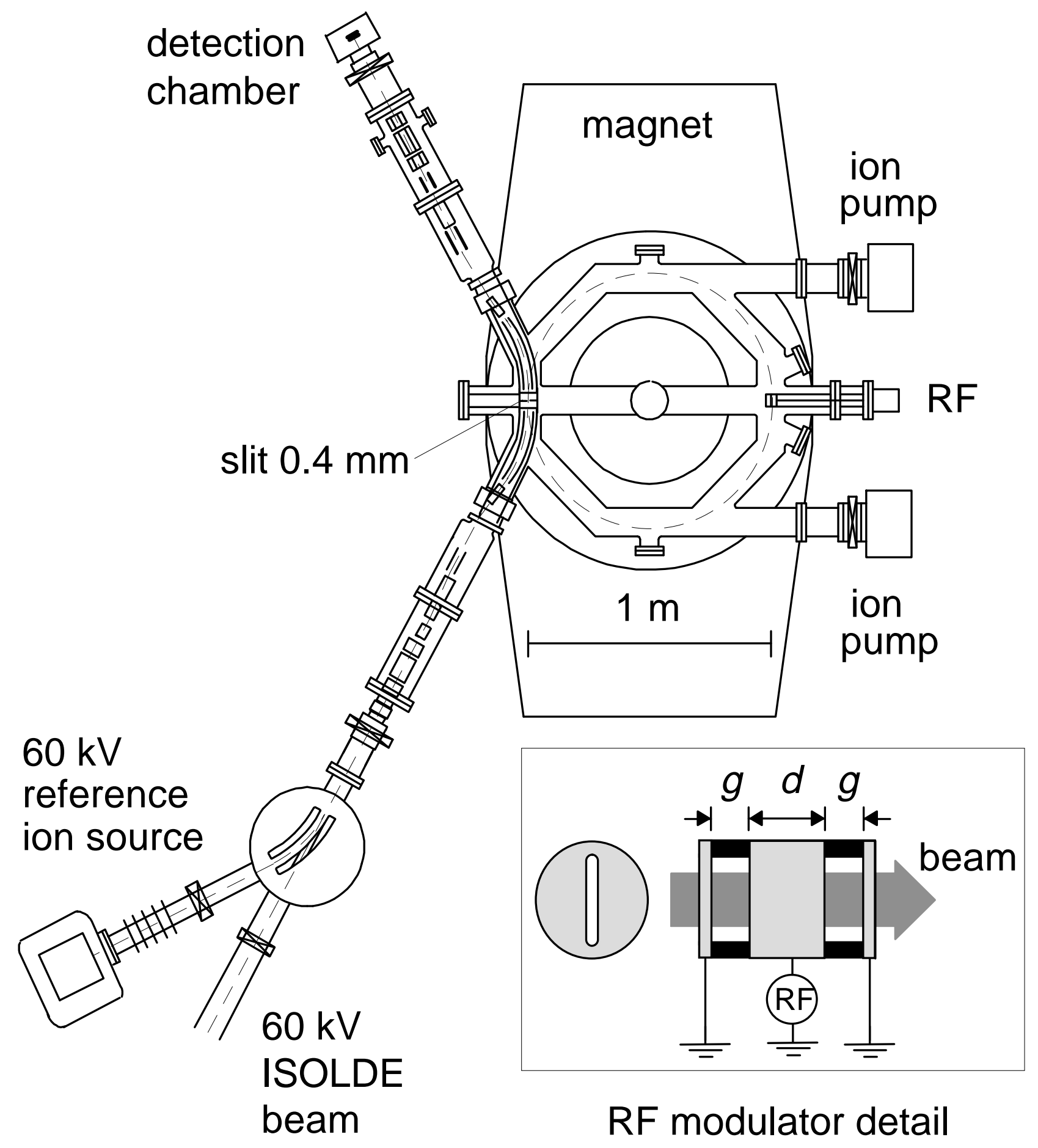




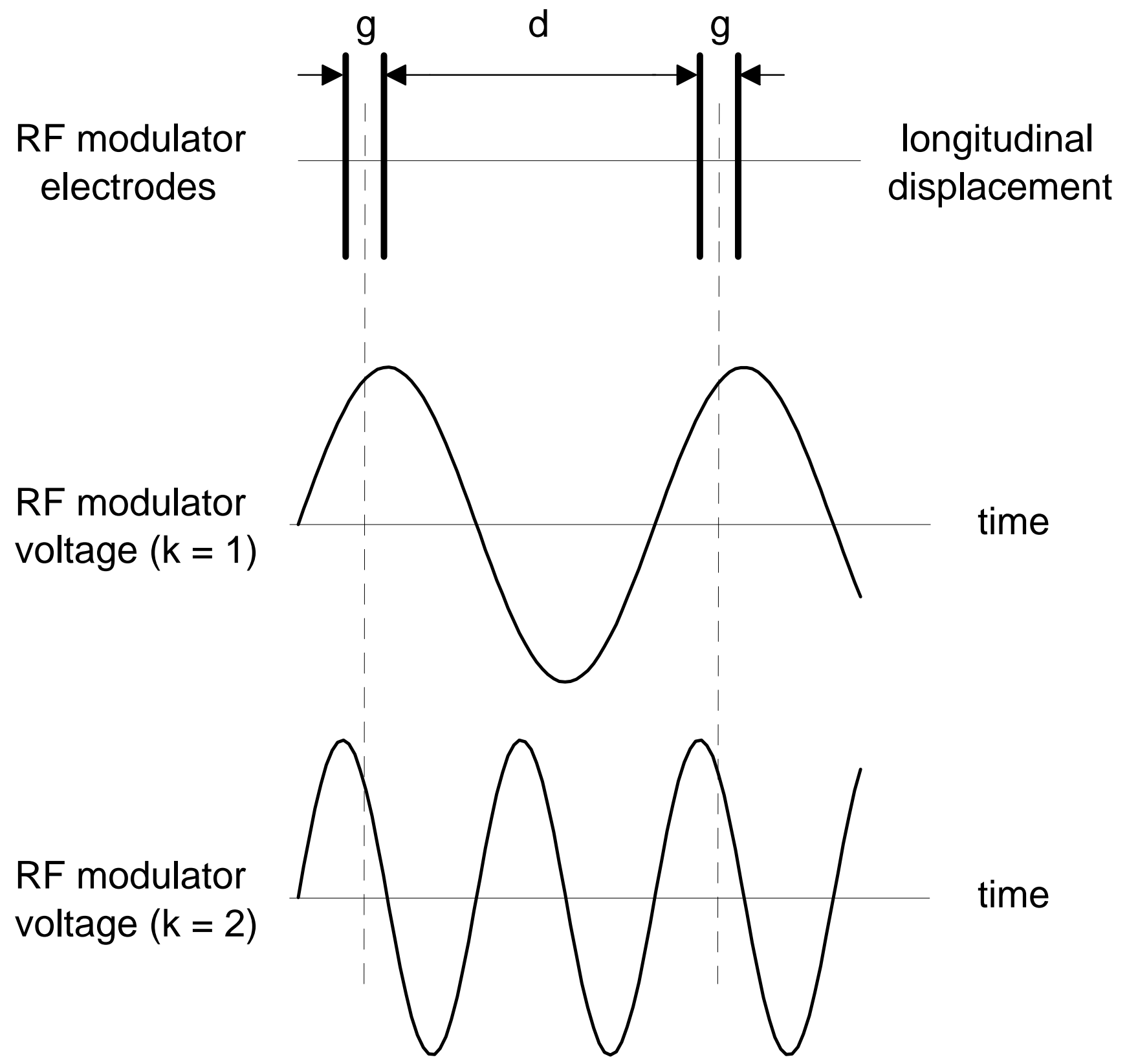

